\title{
Fiber-Optic Quantum Thermometry with Germanium-Vacancy Centers in Diamond Supporting Information
}

\author{
Sean M. Blakley, ${ }^{*}$ Xiaohan Liu, llya Fedotov, Ivan Cojocaru, Christapher \\ Vincent, Masfer Alkahtani, Joe Becker, Michael Kieschnick, Tobias Lühmann, \\ Jan Meijer, Philip R. Hemmer, Alexey V. Akimov, Marlan O. Scully, and Aleksei \\ Zheltikov \\ E-mail: SMB784@TAMU.EDU \\ Pages S1-S3, Figures S1
}




\section{Spectral Fit Procedure}

The susceptibility of fluorescence from region $b$ to changes in temperature was ascertained by fitting the zero-phonon peak of the GeV spectrum with a Lorentzian function (Fig. S1 (a)-(b)) in order to extract the center wavelength and full-width half-maximum (FWHM) of the $602 \mathrm{~nm} \mathrm{GeV} \mathrm{signal} \mathrm{peak} \mathrm{as} \mathrm{a} \mathrm{function} \mathrm{of} \mathrm{temperature.} \mathrm{In} \mathrm{general,} \mathrm{the} \mathrm{most} \mathrm{accurate}$ fit to the spectral data was provided by a double Lorentzian function $L_{2}=\lambda_{w} h /[\pi(\lambda-$ $\left.\left.\lambda_{c}\right)^{2} / \lambda_{w}^{2}+1\right]+\lambda_{w 2} h_{2} /\left[\pi\left(\lambda-\lambda_{c 2}\right)^{2} / \lambda_{w 2}^{2}+1\right]+b$, (Fig. S1 (a) and (b), orange traces), where $\lambda, \lambda_{c}, \lambda_{w}, \lambda_{c 2}, \lambda_{w 2}$ are the spectral wavelength, center wavelength of the first Lorentzian fit peak (Fig. S1), FWHM of the first Lorentzian fit peak, the center wavelength of the second Lorentzian fit peak, and the FWHM of the second Lorentzian fit peak respectively. The parameters $h, h_{2}$, and $b$ correspond to the height of both Lorentzian fit peaks and the offset of the spectral baseline amplitude from zero respectively.

A single Lorentzian function $\left(L_{1}\right)$ provides a somewhat accurate fit for data taken at lower laser powers that remains stable for spectra taken across the entire temperature range (Fig. S1 (a)). The double Lorentzian function sometimes $\left(L_{2}\right)$ fails to accurately fit these noisier spectra, generating errors in the temperature accuracy and resolution calculations for these lower power levels. However, for the higher laser power spectra, the $L_{2}$ fit is much more stable across the entire temperature range than the $L_{1}$ fit (Fig. S1, purple dashed traces), and provides a much more accurate fit, allowing for sensitive temperature measurements.

In order to determine what experimental parameters limit the spatial and temperature resolution of this measurement, the peak amplitudes for spectra taken with 500 ms exposure time (Fig. S1 (c)) were plotted versus laser power (Fig. S1 (d), blue trace). The peak amplitude and signal-to-noise ratio (SNR) were found to depend linearly on laser power (Fig. S1 (d), blue and red traces respectively). The signal-to-noise ratio (SNR) was calculated by dividing the peak amplitude by the maximum noise amplitude at the top of the peak, which is a manifestation of both the detector dark current and laser amplitude fluctuations.

This linear dependence of the SNR and peak amplitude on laser power determined that 

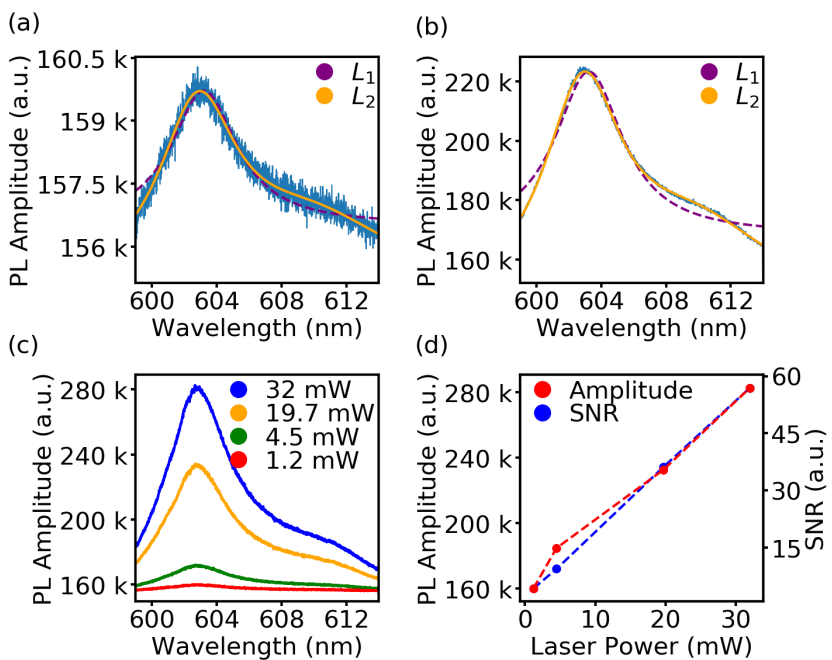

Figure S1: Single and double Lorentzian fit functions (purple, orange traces) for spectra collected with laser powers (a) $1 \mathrm{~mW}$ and (b) $20 \mathrm{~mW}$ at $500 \mathrm{~ms}$ exposure time. (c) Fluorescence spectra taken at $500 \mathrm{~ms}$ exposure time for different power levels. (d) Amplitudes of fluorescence peaks in (c) plotted versus laser power (red trace). SNR as a function of laser power (blue trace) calculated by dividing peak amplitude by maximum amplitude of noise at top of peak.

the $\mathrm{GeV}$ centers in region $b$ were not being saturated by the incident laser light, as a nonlinear dependence of fluorescence amplitude on pump laser power would be expected in that situation. Therefore the fiber core diameter may be reduced until saturation occurs in order to enhance spatial resolution without affecting the collected signal or the SNR. Employing a higher numerical aperture (NA) fiber will enhance the fluorescence collection efficiency, permitting the use of lower laser powers while maintaining signal amplitude, thereby improving SNR by reducing detected thermal noise resulting from laser power fluctuations. 